\title{
Myd88-dependent positioning of Ptgs2- expressing stromal cells maintains colonic epithelial proliferation during injury
}

\author{
Sarah L. Brown, ${ }^{1}$ Terrence E. Riehl,,2 Monica R. Walker, ${ }^{1}$ Michael J. Geske, ${ }^{1}$ \\ Jason M. Doherty, ${ }^{1}$ William F. Stenson, ${ }^{2}$ and Thaddeus S. Stappenbeck ${ }^{1}$
}

${ }^{1}$ Department of Pathology and Immunology and 2Division of Gastroenterology, Washington University School of Medicine, St. Louis, Missouri, USA.

\begin{abstract}
We identified cellular and molecular mechanisms within the stem cell niche that control the activity of colonic epithelial progenitors (ColEPs) during injury. Here, we show that while WT mice maintained ColEP proliferation in the rectum following injury with dextran sodium sulfate, similarly treated $M y d 88^{-/-}$(TLR signaling-deficient) and prostaglandin-endoperoxide synthase $2^{-/-}\left(\right.$Ptgs $\left.^{-/-}\right)$mice exhibited a profound inhibition of epithelial proliferation and cellular organization within rectal crypts. Exogenous addition of 16,16-dimethyl $\mathrm{PGE}_{2}\left(\mathrm{dmPGE}_{2}\right)$ rescued the effects of this injury in both knockout mouse strains, indicating that Myd88 sig-

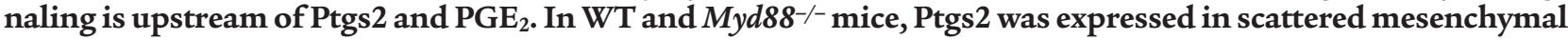
cells. Surprisingly, Ptgs2 expression was not regulated by injury. Rather, in WT mice, the combination of injury and Myd88 signaling led to the repositioning of a subset of the Ptgs2-expressing stromal cells from the mesenchyme surrounding the middle and upper crypts to an area surrounding the crypt base adjacent to ColEPs. These findings demonstrate that Myd88 and prostaglandin signaling pathways interact to preserve epithelial proliferation during injury using what we believe to be a previously undescribed mechanism requiring proper cellular mobilization within the crypt niche.
\end{abstract}

\section{Introduction}

The mammalian intestinal epithelium forms an absorptive barrier that separates a vast and diverse population of luminal microbes (1-3) from the robust capillary networks and immune cell populations located in the underlying mesenchyme of the host (4-5). Disruption or perturbation of this barrier can come from a number of sources, including irradiation as well as infection by pathogenic organisms and cytotoxic chemicals. Because of the unique ecology of the intestine, regulators of barrier maintenance and repair must be deployed in a rapid fashion. A focal point for this process is the proper modulation of colonic epithelial progenitor (ColEP) activity. Our goal in these studies was to determine the roles of the epithelium, the surrounding mesenchymal niche, and various inflammatory cells in making and executing these important decisions in response to a given injury.

The barrier epithelium that lines the inner surface of the mouse cecum and colon is a single layer of nondividing, terminally differentiated cells. These cells undergo constant and rapid replacement during the life of the organism. The source of this renewal is a proliferative population of ColEPs, located in the lower third of epithelial invaginations (called "crypts of Lieberkühn") (6). ColEPs consist of tripotent stem cells that give rise to 2 sets of proliferative committed daughters; monopotent progenitors produce absorptive enterocytes, while bipotent progenitors give

Nonstandard abbreviations used: B6, C57BL/6J; Ccnd1, cyclin D1; ColEP, colonic epithelial progenitor; $\mathrm{C}_{\mathrm{T}}$, threshold cycle; $\Delta \mathrm{C}_{\mathrm{T}}$, change in $\mathrm{C}_{\mathrm{T}} ; \mathrm{dmPGE}_{2}, 16,16$-dimethyl $\mathrm{PGE}_{2}$; DSS, dextran sodium sulfate; Fosl1, fos-like antigen 1; Iigp1, interferon inducible GTPase 1; Indo, indoleamine-pyrrole 2,3 dioxygenase; LCM, laser capture microdissection; Mmp7, matrix metallopeptidase 7; PAS/AB, PAS/Alcian blue; PSC, Ptgs2expressing stromal cell; Ptgs2, prostaglandin-endoperoxide synthase 2; qRT-PCR, quantitative RT-PCR; Reg3g, regenerating islet-derived $3 \gamma$.

Conflict of interest: The authors have declared that no conflict of interest exists. Citation for this article: J. Clin. Invest. 117:258-269 (2007). doi:10.1172/JCI29159. rise to secretory goblet and enteroendocrine cells. All 3 epithelial lineages continue their differentiation program as they migrate upward toward the luminal surface (7). Enterocytes are the predominant cell type that exits the crypt and forms the surface barrier epithelium. Most goblet cells remain in the crypt, and their precise localization within this structure depends on their anatomic location along the length of the colon.

During homeostasis, Wnt and Notch signals are required to maintain ColEP proliferation, while TGF- $\beta$ and bone morphogenetic protein act as key negative regulators. Wnt, Notch, and Hedgehog signaling all play a role in modulating intestinal epithelial cell fate decisions (e.g., refs. 8-12). A key source of many of these signals is considered to be the stem cell niche $(13,14)$. In the colon, the niche is composed of mesenchymal cellular networks of fibroblasts and blood vessels that envelop crypts and project toward the surface epithelium. Most colonic fibroblasts are myofibroblasts that express a subset of smooth muscle-specific proteins and form a prominent anastamosing network of cells that forms a cup-like structure around each crypt (15). These cells may produce a variety of factors that support crypt proliferation and may display an ability to migrate upward toward that luminal surface in concert with the overlying epithelial cells (16). The microvasculature has also been proposed to play a role in ColEP survival during irradiation injury (17). The role of nonmyogenic fibroblasts in the crypt-associated mesenchyme is unclear.

During certain types of injury, the crypt base niche requires a cellular adaptation from the immune system to properly support the overlying epithelial response that is required for the overall health of the animal. In a chemical injury model involving oral introduction of dextran sodium sulfate (DSS), mice that are deficient for either indigenous colonic microbes or TLR signaling have diminished survival and more severe weight loss because ColEPs no longer divide at an appropriate rate to properly maintain the epithelial barrier 


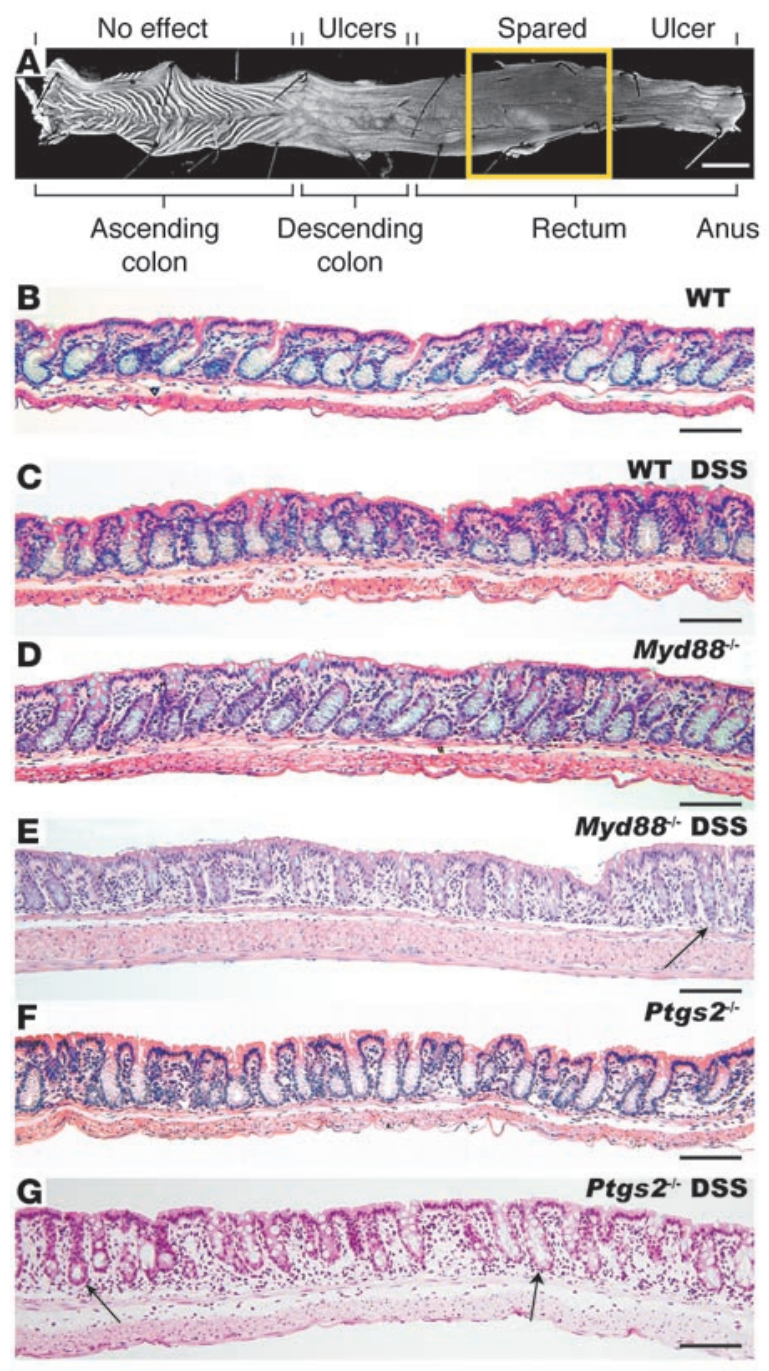

(18-21). In a previous study, we found that macrophages, activated by TLR signaling, are recruited to the colonic stem cell niche and are required for the proper epithelial response to this injury (18).

Our previous microarray experiments using laser capture microdissected cells from the pericryptal mesenchyme surrounding hyperproliferative crypts and hypoproliferative crypts from DSS-injured mice identified prostaglandin-endoperoxide synthase 2 (Ptgs2 or Cox-2) as a candidate mediator of the ColEP response (18). In the intestine, Ptgs2 is expressed in a small subset of lamina propria stromal cells, while its homolog, Ptgs1, is expressed primarily in the crypt epithelium and lamina propria mononuclear cells (22-24). Together, they play a vital role in the production of prostaglandins that can have both growth-stimulatory and antiapoptotic effects (25). Ptgs1 and -2 have also been shown to play a significant role in protection of the intestine from both irradiation and DSS-mediated damage $(24,26$, 27), primarily though the production of $\mathrm{PGE}_{2}$. Recently $\mathrm{PGE}_{2}$ was shown to transactivate the canonical Wnt signaling pathway (28).

Because signaling through Myd88-dependent TLRs and Ptgs2 (through $\mathrm{PGE}_{2}$ ) play similar roles in limiting gut injury to a variety of stimuli, we hypothesized that these pathways might be either coordinated or linked during the injury response. In this study, we evaluated the role of Ptgs 2 and its relationship with Myd88 sig-

\section{Figure 1}

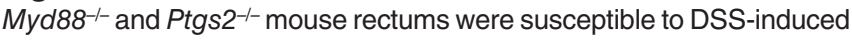
injury. (A) Colon whole mount from an adult WT B6 mouse treated with $2.5 \%$ DSS for 1 week. The DSS-induced lesions (top) and the corresponding anatomic locations (bottom) are indicated. All strains analyzed in this study showed multiple small $(<0.5 \mathrm{~mm}$ in diameter) ulcers with heaped borders in the descending colon and a confluent ulcer that extended from the ano-rectal junction proximally $(0.5-1.0 \mathrm{~cm})$. The intervening area in the remaining rectum (yellow boxed area) was spared of ulcerative injury. (B-G) H\&E-stained sections of the rectums

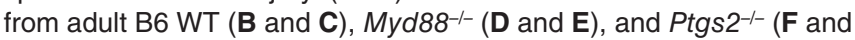
G) mice. (B, D, and F) Untreated mice. (C, E, and G) Mice treated for 7 days with $2.5 \%$ DSS in the drinking water. The rectums from WT DSStreated mice were indistinguishable from those of untreated WT mice. However, DSS-treated $\mathrm{Myd}_{88^{-/}}$and $\mathrm{Ptgs} 2^{-/-}$mice both showed a similar pattern of crypt area loss manifested by alterations in crypt morphology (including angulation and dilation of the base; arrows) as compared with untreated controls. Scale bars: $1 \mathrm{~cm}(\mathbf{A}), 100 \mu \mathrm{m}(\mathbf{B}-\mathbf{G})$.

naling in mice injured with DSS. We found that absence of either Myd88 or Ptgs2 in DSS-treated mice profoundly inhibited ColEP proliferation in the mouse rectum. We found that exogenous 16,16-dimethyl PGE (dmPGE $\left._{2}\right)$ rescued this effect in both knockout mice, establishing that Ptgs 2 and $\mathrm{PGE}_{2}$ were downstream of Myd88 signaling. Surprisingly, Myd88 did not modulate Ptgs2 expression, but instead altered the positioning of Ptgs2-expressing stromal cells (PSCs) in the lamina propria mesenchyme relative to ColEPs in the crypt base. This represents what we believe to be a novel finding of a cellular niche modification that is required to maintain colonic epithelial proliferation in response to injury.

\section{Results}

The crypt epithelial response to DSS-mediated rectal injury was similar

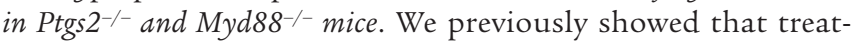
ment of WT adult, male C57BL/6J (B6) mice with $2.5 \%$ DSS for 1 week resulted in colonic injury in the form of focal ulcers in the descending colon and a single confluent ulcer at the ano-rectal junction (18) (Figure 1A). The crypts surrounding the ulcers in the descending colon were elongated and contained an expansion of ColEPs. This process depended on the presence of microbes in the lumen, detection during injury by host bone marrow-derived cells through Myd88-dependent TLR signaling, and presence of activated macrophages in the crypt base niche (18). In contrast, the rectal tissue between these major injury sites was not severely injured in DSS-treated WT mice (Figure 1A, boxed area). By whole mount analysis, the mucosal surface of this area was uniform and did not contain ulcers $(n=20)$. Low-power histologic analysis of this area of the rectum showed that the cellularity of the mesenchyme and the density and architecture of crypt-surface units was not perceptibly altered when similar regions of untreated and DSS-treated WT mice were compared (Figure 1, B and C).

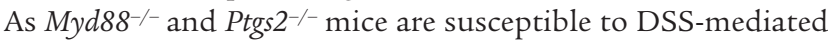
damage in the colon $(18-21,26)$ and $\mathrm{PGE}_{2}$ can play a role in the amelioration of DSS-induced damage in WT mice (24), we hypothesized that a mechanistic link exists between Myd88 and Ptgs2 signaling pathways that protects the colonic mucosa from this type of damage. We evaluated the response to DSS-mediated injury in the rectums of $\mathrm{Myd}_{88^{-/-}}$and Ptgs $2^{-/-}$mice using our experimental protocol. Low-power histologic views of the rectums from untreat-

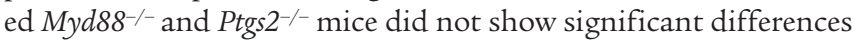
in the morphology of crypt-surface epithelial units and the overall 

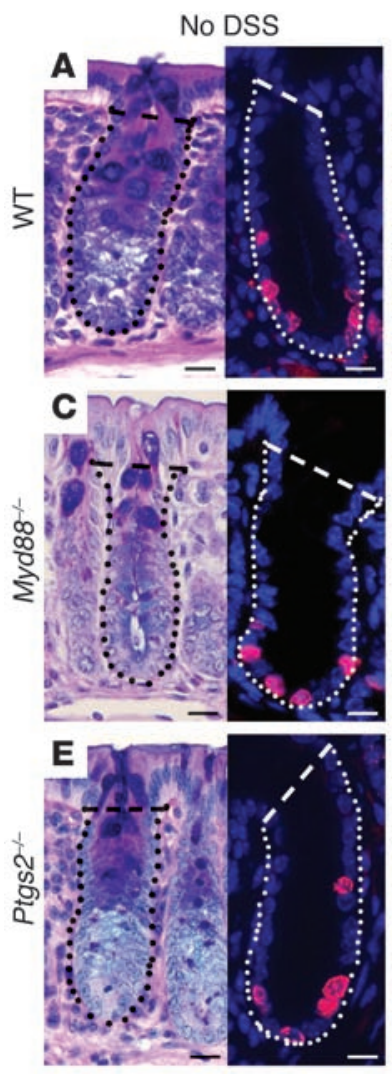
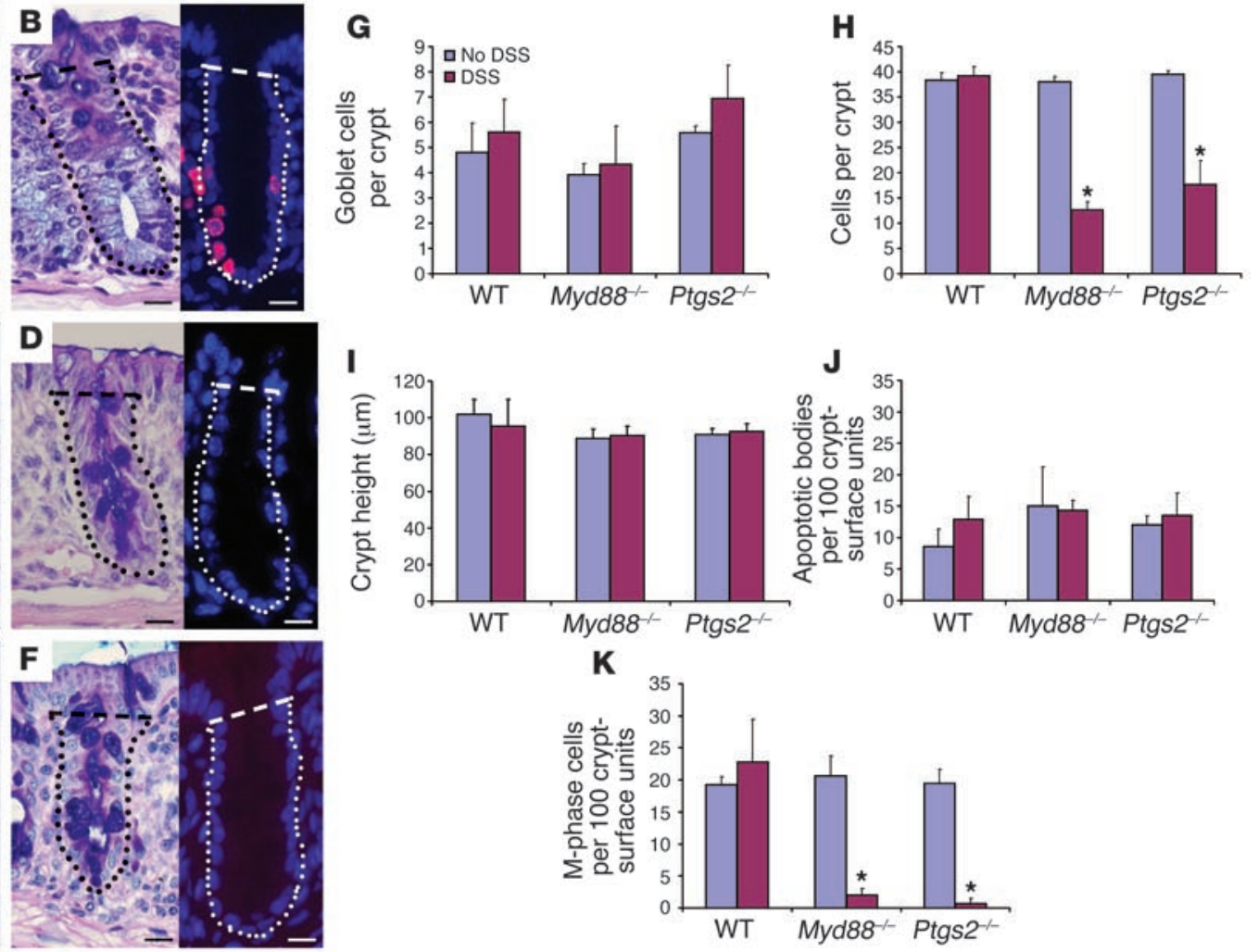

Figure 2

Goblet cell reorganization and loss of epithelial proliferation in the rectum of DSS-treated Ptgs $2^{-/-}$and $M y d 88^{-/-}$mice. (A-F) Sections of a rectal

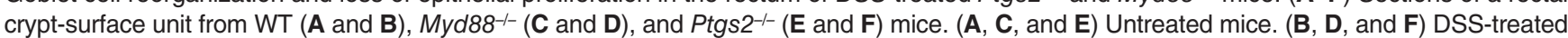
mice. Sections were stained with PAS/AB to identify goblet cells (left) or with goat anti-BrdU, Alexa-Fluor 594-labeled donkey anti-goat Ig (red), and bis-benzimide (blue nuclear stain) to identify cells in S-phase (right). Scale bars: $20 \mu \mathrm{m}$. The crypt epithelial-mesenchymal (dotted lines) and the epithelial crypt-surface junctions (dashed lines) are indicated. Quantification of goblet cells per crypt (G), crypt cell census (H), crypt height (I), epithelial apoptosis $(\mathbf{J})$, and epithelial proliferation $(\mathbf{K})$. Mean values \pm SEM were plotted for each group. An asterisk indicates a value that is statistically significantly different from the corresponding untreated control ( ${ }^{*} P<0.001$; Student's $t$ test). Both DSS-treated $\mathrm{Myd} \mathrm{B} 8^{-/-}$and $P$ tgs $2^{-/-}$mice showed a statistically significant decrease in epithelial proliferation and crypt cell census as compared with their corresponding untreated controls.

cellularity of the underlying lamina propria mesenchyme as compared with WT mice (Figure 1, B, D, and F).

The gross appearance of whole mount preparations of the colons from DSS-treated $\mathrm{Myd}_{88^{-/-}}$and Ptgs2 $2^{--}$mice showed an overall pattern of injury similar to that in WT DSS-treated mice. Ulcers were observed in the descending colon and ano-rectal junction, while the intervening rectal mucosa of both knockout strains was ulcerfree (data not shown). However, low-power histologic views of the

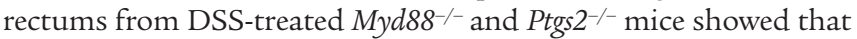
crypts were much less prominent as compared with those in rectums from similarly treated WT mice and their untreated counterparts. While the number of crypts per unit area was not altered in the mice, each sectioned crypt contained less surface area (corresponding to less volume in 3 dimensions). Crypt morphology was typically altered to increase either lumen size or angulation of the base (Figure 1, E and G). The loss of crypt volume corresponded to an increase in the

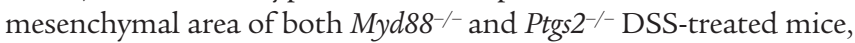
though the overall cellular density in this region was not perceptibly altered. These effects were specific for the absence of Ptgs2, as rectums from DSS-treated Ptgs $1^{-/-}$mice had the same overall appearance as those from WT DSS-treated mice (data not shown).
Both Ptgs 2 and Myd88 were required to maintain crypt organization and epithelial proliferation during DSS-mediated injury. Because rectal crypt alterations were prominent in DSS-treated $\mathrm{Myd}_{88^{-/-}}$and Ptgs2 $2^{--}$ mice, we evaluated goblet cells and ColEPs, the 2 major cell types in this microanatomic compartment. To visualize goblet cells, we used a pan-mucin stain that labels both neutral and acidic mucosubstances (PAS/Alcian blue [PAS/AB] stain) (29). Fully differentiated goblet cells (containing a single large apical theca) were located primarily in the upper half of rectal crypts from untreated WT mice. The lower half of crypts consisted primarily of ColEPs that showed scant, diffuse PAS/AB cytoplasmic staining. A subset of the ColEPs showed focal staining in smaller apical thecae, consistent with committed goblet cell progenitors (Figure 2A) (6). The overall distribution of goblet cells and ColEPs was not perceptibly altered in either

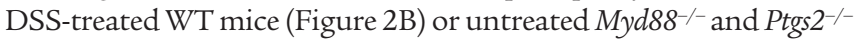
mice (Figure 2, C and E). However, goblet cells in rectal crypts of

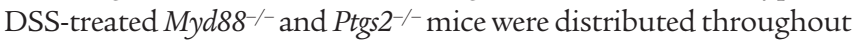
rectal crypts (Figure 2, D and F).

Because goblet cells appeared to be a more prominent component

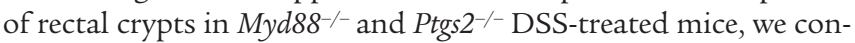
sidered that epithelial differentiation was altered to preferentially 


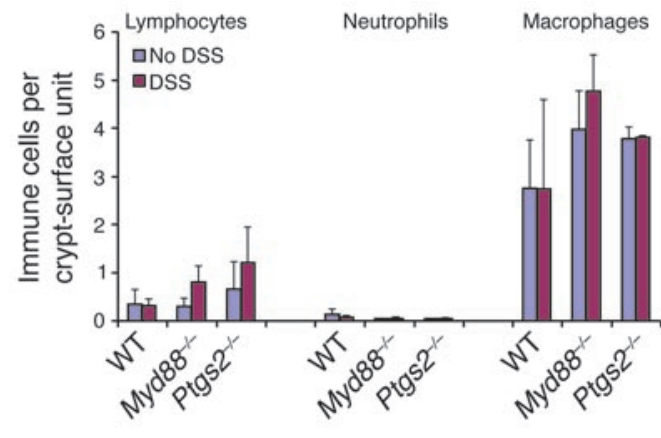

Figure 3

There was no statistically significant inflammatory infiltrate in the rectum of DSS-treated mice. Quantification of lymphocytes (B220 and CD3ع), neutrophils and eosinophils (Gr-1), and macrophages (F4/80) per cryptsurface unit. Mean values \pm SEM were plotted for each group. No statistically significant differences were observed when comparing any of the DSS-treated mice with untreated mice (Student's $t$ test; $P<0.05$ ).

favor selection of secretory lineages, perhaps through an inhibition of Notch signaling (9). However, the number of goblet cells per crypt was not altered either by genotype or by DSS-mediated injury (Figure 2G). Goblet cells appeared to be more prominent, as rectal

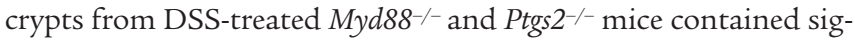
nificantly fewer total cells per crypt (Figure 2H; each DSS-treated knockout mouse contained less than half the cells of its untreated counterpart; $P<0.001 ; n=3-5$ mice/group, 100 well-oriented rectal crypts evaluated/mouse; "well-oriented" was defined as the crypt lumen being present from the top to the bottom of the crypt), thus increasing the fractional representation of this lineage. Interestingly, although $\mathrm{Myd} 88^{-/-}$and Ptgs $2^{-/-}$rectal crypts contained fewer cells, they were not significantly shortened as a result of DSS treatment (Figure 2I). Thus, alterations in overall crypt morphology and individual cells created the striking images that defined the phenotype at low-power histology.

The loss of cells within rectal crypts of DSS-treated $M y d 88^{-/-}$and Ptgs $2^{-/-}$mice could have resulted from diminished epithelial input (proliferation) and/or increased outflow (cell death). Therefore, we scored for proliferation by quantifying M-phase cells per crypt and for cell death by quantifying apoptotic bodies per crypt-surface unit within the epithelium. We found no significant differences in epithelial cell death when comparing untreated and DSStreated mice of any genotype (Figure 2J). We obtained the same results when we evaluated the crypt and the surface epithelium separately (data not shown). However, quantification of M-phase cells per crypt showed that Ptgs2 $2^{-/}$and $M y d 88^{-/-}$mice treated with DSS showed a significant decrease in epithelial proliferation when compared with their untreated counterparts (Figure 2K; proliferation in each knockout was less than 10-fold that of its untreated counterpart; $P<0.001 ; n=3-5$ mice/group, 100 well-oriented rectal crypt-surface units evaluated/mouse). In support of this finding, S-phase cells located in the lower third of normal crypts were nearly absent in DSS-treated Ptgs $2^{-/-}$and $\mathrm{Myd} 88^{-/-}$mice (Figure 2, A-F). Thus, a major target of the DSS-mediated effects was the proliferative activity of the ColEPs.

No significant inflammatory infiltrates occurred in the rectum of DSStreated mice. Previously, we found that the lamina propria mesenchyme underlying crypts that bordered ulcers in WT DSS-treated mice showed a substantial influx of inflammatory cells, including neutrophils, lymphocytes, and macrophages (18). Similar areas of DSS-treated $M y d 88^{-1-}$ mice showed no significant increase in lymphocytes and neutrophils. The influx of macrophages was similar to that in WT mice, though the macrophages were not activated either morphologically or molecularly in the absence of Myd88. In contrast, the rectal mesenchyme of WT DSS-treated mice showed no statistically significant alteration in immune cell populations.

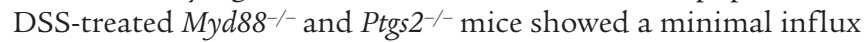
of lymphocytes and neutrophils (not statistically significant) and no increase in macrophages in the rectum (Figure 3; $n=3-5$ mice evaluated/group; lamina propria mesenchyme associated with 300 well-oriented perpendicular sections through crypt-surface units/ mouse). We concluded that the near ablation of rectal epithelial

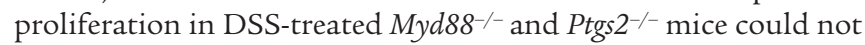
be explained simply as a response to inflammation.

Exogenous dmPGE $E_{2}$ rescued the DSS-mediated rectal injury in both

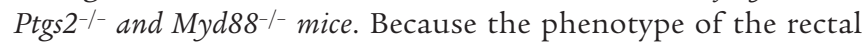
mucosa of DSS-injured Myd88 ${ }^{-/-}$and Ptgs $2^{-/-}$mice was similar by all quantitative morphometric parameters, we tested the hypothesis that these genes functioned in the same genetic pathway, with Ptgs2 downstream of Myd88. We attempted to rescue the phenotype of DSS-treated Myd88 $8^{-/}$mice with a key prostaglandin produced in the intestine $\left(\mathrm{PGE}_{2}\right)$ that is regulated in part by Ptgs 2 (24). For in vivo administration, we used $\mathrm{dmPGE}_{2}$, which has previously been utilized and exhibits a longer half-life compared with endogenous $\mathrm{PGE}_{2}(23)$.

We evaluated multiple doses of $\mathrm{dmPGE}_{2}$ in DSS-treated WT and Ptgs $2^{-/-}$mice. Injection of $10 \mu \mathrm{g} \mathrm{dmPGE} / 2 / \mathrm{kg}$ body weight twice per day during the 7 days DSS treatment did not alter the rectal mucosal morphology of WT DSS-treated mice by any of the previously described morphometric parameters (Figure 4A). Higher doses of $\mathrm{dmPGE}_{2}$ partially inhibited rectal epithelial proliferation in WT DSS-treated mice (data not shown). The treatment regimen of $\mathrm{dmPGE}_{2}$, optimized for WT DSS-treated mice, completely rescued the crypt alterations (dysmorphic crypts and goblet cell disorganization) that occurred in DSS-treated Ptgs $2^{--}$mice (Figure 4B). Quantification of key aspects associated with the phenotype showed that epithelial proliferation and the crypt census occurred at levels comparable to those in WT DSS-treated mice (Figure 4, $\mathrm{D}$ and $\mathrm{F} ; P<0.001 ; n=5$ mice/group).

The same regimen of $\mathrm{dmPGE}_{2}$ also rescued the rectal phenotype

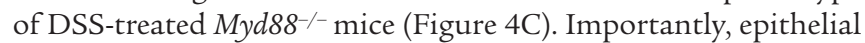
proliferation and crypt census in these mice were significantly different from those in controls (DSS-treated $M y d 88^{-1-}$ mice in the absence of exogenous $\mathrm{dmPGE}_{2}$ ) and were comparable to those in DSS-treated WT and Ptgs $2^{-/-}$mice with the addition of this drug (Figure 4, D and F; $P<0.001 ; n=5$ mice/group). Parameters not previously altered by DSS treatment, including apoptosis (Figure 4E), goblet cell number, and crypt height (data not shown), were also unaltered by the addition of $\mathrm{dmPGE}_{2}$.

Ptgs 2 mRA transcripts were expressed in the mesenchyme but were not increased by injury or Myd88 status. The rescue of DSS-treated

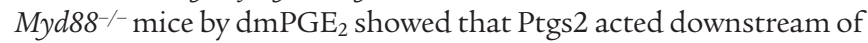
Myd88. Our goal was to discern the precise relationship between these 2 genes that required both to prevent the loss of rectal ColEP proliferation during DSS injury. Our initial hypothesis was that Myd88 signaling regulated Ptgs2 expression.

To test this idea, we established a quantitative assay for the microanatomic localization of Ptgs 2 transcripts. We used laser capture microdissection (LCM) to separately procure rectal mesenchymal 

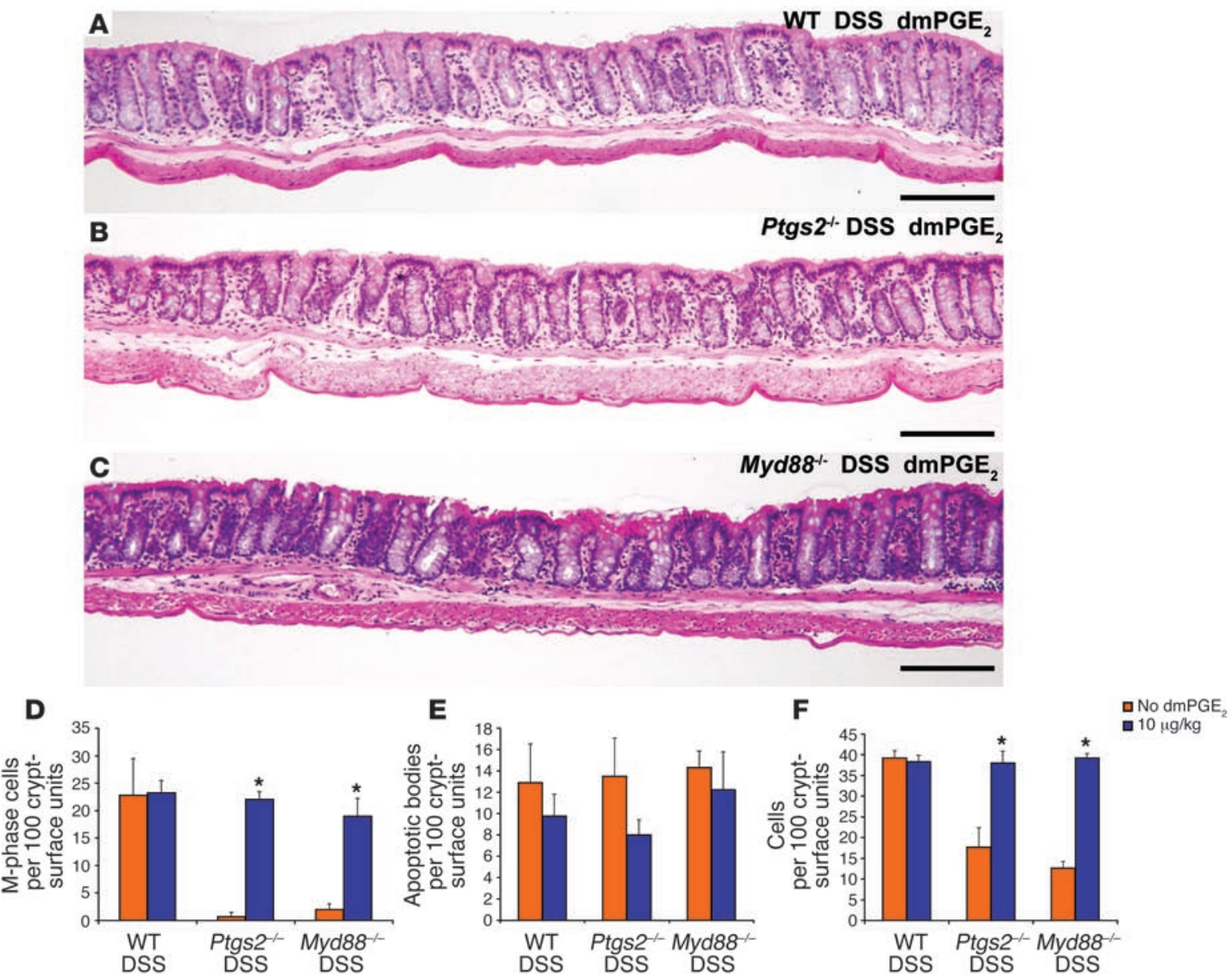

Figure 4

Exogenous dmPGE ${ }_{2}$ rescued the rectal phenotype of DSS-treated Ptgs2 ${ }^{-/-}$and $M y d 88^{-/-}$mice. (A-C) H\&E-stained sections of rectums from (A)

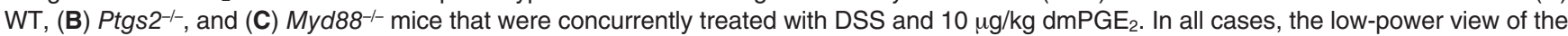
rectum was similar to that of WT DSS-treated mice in the absence of exogenous dmPGE 2 (Figure 1C). Scale bars: $100 \mu \mathrm{m}$. (D-F) Quantification of (D) epithelial proliferation, $(E)$ epithelial apoptosis, and (F) crypt cell census. Mean values \pm SEM were plotted for each group. An asterisk indicates a value that is statistically significantly different from the corresponding control that did not receive $\mathrm{dmPGE}_{2}\left({ }^{*} P<0.001\right.$; Student's $t$ test). This dosage of dmPGE 2 did not affect the rectum of WT DSS-treated mice and rescued the rectal phenotype of DSS-treated $P$ tgs $2^{-/-}$and $M y d 88^{-/-}$mice.

lamina propria and epithelial cells (both crypt and surface cells together) for quantitative RT-PCR (qRT-PCR) assays that compared Ptgs 2 transcript levels in these 2 cellular compartments. Total RNA was isolated from each cellular compartment from 4 experimental groups (WT and $M y d 88^{-/-}$both with and without DSS treatment). The resulting cDNAs were used for SYBR Green-based qPCR detection of 10 genes. For each gene, the threshold cycle $\left(C_{T}\right)$ for each amplicon was subtracted from a baseline (18S rRNA) to calculate a change in $\mathrm{C}_{\mathrm{T}}\left(\Delta \mathrm{C}_{\mathrm{T}}\right)$. For pair-wise comparisons, the mesenchymal $\Delta \mathrm{C}_{\mathrm{T}}$ (numerator) was divided by its corresponding epithelial $\Delta \mathrm{C}_{\mathrm{T}}$ (denominator) to calculate a fold difference.

As a control for the relative transcript enrichment within a given compartment, we used the same cDNAs to analyze transcripts that are preferentially expressed in the mesenchyme (vimentin) and epithelium (E-cadherin) as previously described (18, 30, 31). Mesenchymal dissections contained a 9-fold enrichment of vimentin transcripts as compared with material from epithelial dissections performed on the same sections (Figure $5 \mathrm{~A} ; n=12$ mice), while epithelial dissections contained a 5-fold enrichment of E-cad- herin transcripts as compared with the mesenchyme (Figure 5A). These data were pooled from comparisons using material from all 4 experimental groups (WT and $M y d 88^{-/-}$both with and without DSS treatment). Each individual group contained comparable levels of enrichment of vimentin and E-cadherin (data not shown).

We used the enrichment of E-cadherin transcripts in the epithelium and vimentin transcripts in the mesenchyme as benchmarks to compare the localization of other transcripts. We performed additional qRT-PCR analysis using the same set of microdissected material for markers of mesenchymal cells that might express Ptgs2 (e.g., F4/80 for macrophages; CD11c for dendritic cells; Pecam for endothelial cells). Each of these cellular markers was enriched in the mesenchyme (similar to vimentin) for all experimental groups (a mean of 4.5- to 7-fold; Figure 5A). Each of the 4 experimental groups contained similar levels of transcript enrichment in the mesenchyme (data not shown).

Using the same microdissected material that was used for the dissection controls, qRT-PCR analysis showed that Ptgs 2 transcripts were elevated in the mesenchyme 5-fold, a level of enrichment com- 
A

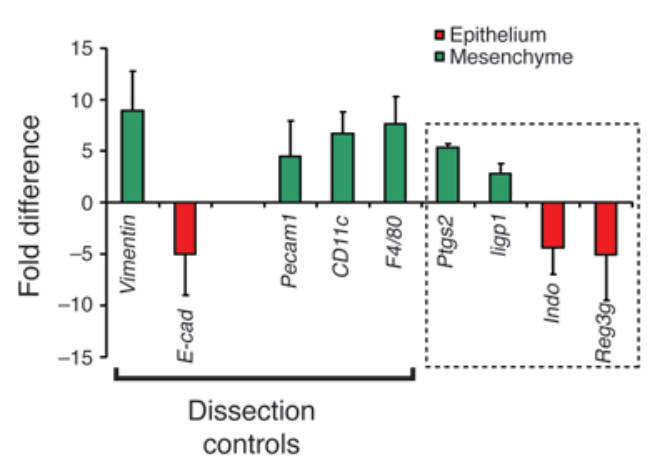

B

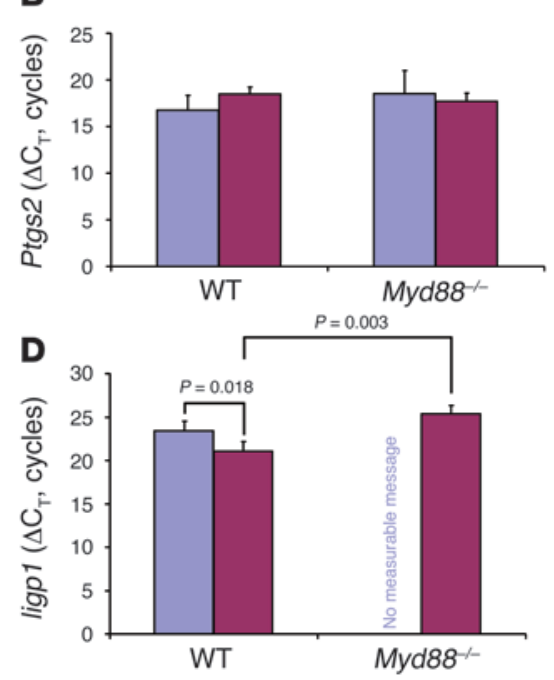

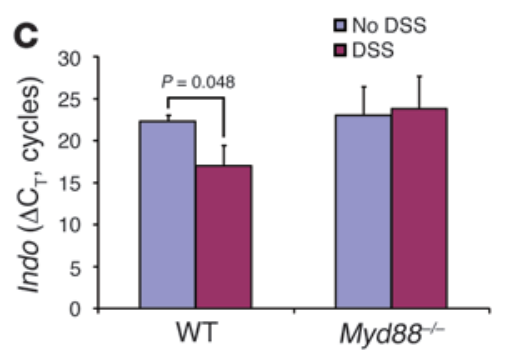

E

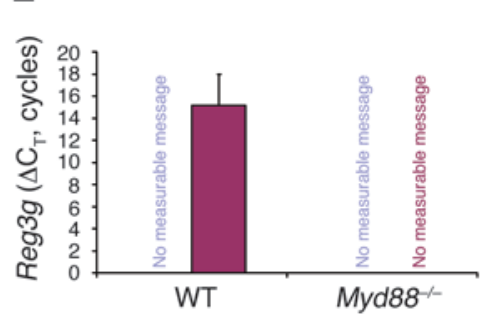

Figure 5

Ptgs2 transcripts expressed in the lamina propria mesenchyme were not elevated in response to injury. (A) qRT-PCR analysis of LCM-procured mRNAs from the rectal mucosal mesenchyme versus the overlying epithelium. A positive value (green) indicated transcript enrichment in the mesenchyme, and a negative value (red) indicated enrichment in the epithelium. The SEM of the fold difference for each gene was calculated from the averages of each experimental group analyzed (WT and $M y d 88^{-/-}$mice with and without DSS treatment). Dissection controls included vimentin, Pecam1, CD11C, and F4/80 for the mesenchyme and E-cadherin (E-cad) for the epithelium. Ptgs2, ligp1, Indo, and Reg3g were identified in our prior microarray screens for transcripts elevated with DSS injury (boxed region). Ptgs2 and ligp1 were enriched in the mesenchyme, while Indo and Reg $3 g$ were enriched in the epithelium. (B-E) Shown are mean \pm SD of $\Delta \mathrm{C}_{\mathrm{TS}}$ for Ptgs2 (B), Indo (C), ligp1 (D), and Reg3g (E) as measured from the compartment where the transcript was enriched. A smaller $\Delta \mathrm{C}_{\mathrm{T}}$ indicates greater expression. The relative transcript levels for Ptgs 2 were not significantly different between DSS-treated and untreated controls for either WT or Myd88 ${ }^{-/-}$rectal mesenchyme. Both ligp1 and Indo were significantly enriched in DSS-treated WT mice (versus untreated). Signal for Reg3g was only detected in the WT DSS-treated sample.

parable to that of all of the other mesenchymal markers (Figure 5A), suggesting this was the primary microanatomic site of Ptgs 2 expression. As a control, material from a Ptgs $2^{-/-}$mouse was used to obtain a null signal (data not shown). We also evaluated other genes we previously identified as enriched in WT DSS-treated mice relative to Myd88-1- DSS-treated mice (18). We found that transcripts for an interferon inducible GTPase 1 (Iigp1) were also enriched in the mesenchyme while indoleamine-pyrrole 2,3 dioxygenase (Indo) and regenerating islet-derived $3 \gamma(\operatorname{Reg} 3 g)$ were preferentially expressed in the epithelium using the exact same set of microdissected material (Figure 5A).

We then determined the regulation of mesenchymal Ptgs 2 transcript abundance by making 3 separate pair-wise comparisons between (a) untreated and DSS-treated WT mice, (b) untreated and DSS-treated Myd88-/- mice, and (c) DSS-treated WT and

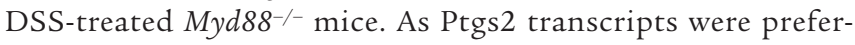
entially located in the mesenchyme, we performed these comparisons using material from this microanatomic compartment. Surprisingly, no statistically significant differences in Ptgs 2 transcript abundance were found for any of these 3 comparisons (Figure 5B; $n=3$ mice/group). Therefore, neither the status of injury nor Myd88 affected Ptgs2 expression in this system.

As a control for the injury response in WT versus $M y d 88^{-/-}$rectums, we evaluated transcript levels of Iigp1, Indo, and Reg3g using exactly the same set of LCM-procured samples and the same set of pair-wise comparisons. In contrast to Ptgs2, all 3 of these transcripts showed significant Myd88- and injury-dependent alterations in transcript abundance in the microanatomic compartment where they were preferentially expressed. In the mesenchyme, Iigp 1 was enriched (smaller $\Delta \mathrm{C}_{\mathrm{T}}$ ) in WT DSS-treated mice as compared with
WT untreated (Figure 5D; 5-fold difference; $P<0.05$ ) and Myd88 DSS-treated mice (19-fold difference; $P<0.05)$. Similar enrichments were observed with the 2 epithelially expressed genes (Indo and Reg3g). Transcripts for Indo were enriched in WT DSS-treated mice as compared with WT untreated mice (Figure 5C; 39-fold difference; $P<0.05)$. Reg $3 g$ was an extreme case because transcripts were only detected in the WT DSS-treated samples, and thus comparative calculated comparisons could not be performed (Figure 5E).

Myd88 signaling redistributed the location of PSCs toward the crypt base epithelium. Because Ptgs $2 \mathrm{mRNA}$ levels were not altered in response to DSS-mediated injury, we evaluated the cellular origin and quantified the location of PSCs by examining immunohistochemically

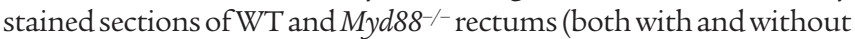
DSS treatment) with antibodies directed against Ptgs2. In all cases, we found scattered, elongated Ptgs2-positive-stained cells restricted to the lamina propria mesenchyme (e.g., WT DSS; Figure 6). Occasional 2-cell clusters were observed. Similarly stained rectums from Ptgs $2^{-/}$mice showed no staining (data not shown).

PSCs were previously identified in the normal small intestine (22) and are increased by irradiation injury and early adenoma formation $(23,32)$. However, no definitive cell-surface markers have been identified to characterize these cells. We found that PSCs in both untreated and DSS-treated WT and $M y d 88^{-/-}$mice showed robust staining for $\mathrm{CD} 44$ (Figure $6, \mathrm{~A}-\mathrm{C} ; n=3$ mice/group). As this surface marker can be expressed by a variety of immune and fibroblast cell types, we attempted to further characterize this cell type. We found variable weak to absent staining of PSCs with the general leukocyte marker CD45 (Figure 6, D-F) in all experimental groups. However, PSCs were uniformly negative for all of the well- 

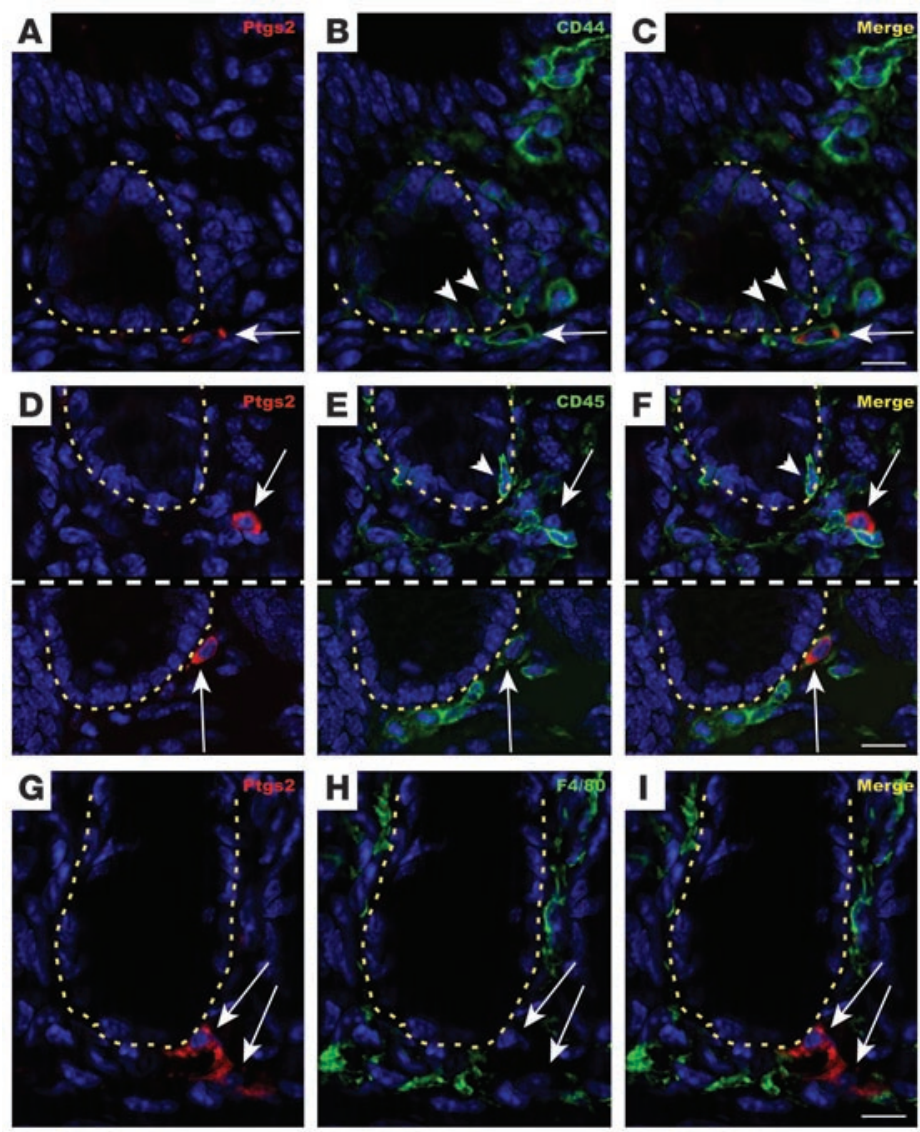

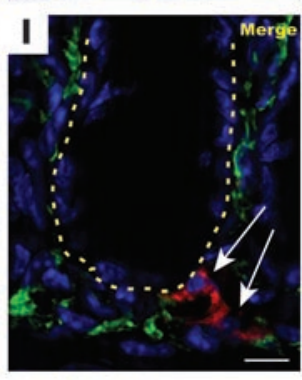

\section{Figure 6}

PSCs are CD44+ stromal cells. (A-I) Double-labeled, immunofluorescence-stained rectal sections from a WT DSStreated mouse. (A-C) Sections stained with Zenon Alexa Fluor 594-labeled anti-Ptgs2 Ig (Invitrogen) (PSCs, red), FITC-labeled anti-CD44 lg (green), and bis-benzimide (blue). All PSCs show membrane staining for CD44. Arrowheads denote CD44+ epithelial cells in the crypt base. (A) Ptgs2, (B) CD44, and (C) merged image of $\mathbf{A}$ and $\mathbf{B}$. (D-F) Two crypt bases from a section stained with Zenon Alexa Fluor 594-labeled anti-Ptgs2 Ig (PSCs, red) and FITC-labeled anti-CD45 Ig (leukocytes, green). (D) Ptgs2, (E) CD45, and (F) merged images (upper panel shows a CD45- PSC, and lower panel shows a weakly CD45+ PSC). The arrowhead denotes the position of a CD45+ intraepithelial lymphocyte. (G-I) Section stained with (G) Zenon Alexa Fluor 594-labeled anti-Ptgs2 Ig (PSCs) and (H) FITC-labeled anti-F4/80 Ig (macrophages, green). (I) Merged image of $\mathbf{G}$ and H. The PSCs in the rectal mesenchyme did not colocalize with this or any other marker of differentiated hematopoietic cell lineages. In all panels, the arrows indicate the position of PSCs, and the yellow dashed lines indicate the crypt epithelial base. Scale bars: $20 \mu \mathrm{m}$. characterized hematopoietic lineage markers, including F4/80 (macrophages; Figure 6, G-I), CD11c (DCs), CD11b, B220 (B cells), CD3e (T cells), NK1.1 (B6 NK cells), Gr-1 (neutrophils and eosinophils), c-Kit (mast cells), and CD70 (data not shown) (33). In addition, markers for fibroblasts/myofibroblasts also failed to show colocalization with PSCs, including anti- $\alpha$-SMA (Figure 7 , A-D), laminin, propyl 4-hydroxylase, and fibroblast-specific protein 1 (data not shown). PSCs also did not express endothelial (Pecam1) or ganglion cell (neuron $\beta$-tubulin) markers under any of the experimental conditions. Thus, we define PSCs as CD44+, differentiated hematopoietic lineage-negative, myofibroblast lineage-negative cells and interpret these as a subpopulation that has not been previously characterized in the mouse colon.

Quantitative analysis showed that under all experimental conditions used in this study, the number of PSCs per crypt-surface unit was approximately 25 -fold lower than F4/80-positive macrophages. In addition, no statistically significant differences in the number of PSCs were found when we compared untreated with DSS-treated mice (both WT and Myd88---; Figure 7E; $n=6-8$ mice/group).

Because Ptgs 2 transcript abundance and the number of PSCs were not altered by injury or Myd88 signaling, we measured $\mathrm{PGE}_{2}$ levels in the entire rectum (Table 1). We found that the levels of this key prostaglandin were not significantly altered in WT mice that were treated with DSS (see ref. 24 for examples of significant alterations). Also, DSS-treated $M y d 88^{-{ }^{-}}$and Ptgs $2^{-{ }^{--}}$mouse rectums both contained levels of $\mathrm{PGE}_{2}$ similar to those in WT DSStreated mice. This is further evidence that neither Ptgs 2 expression nor its activity was dependent on injury or Myd88 gene status.
We observed that most PSCs were localized near the crypt base in WT DSS-treated mice, while other crypt niche constituents such as myofibroblasts were not noticeably reorganized (Figure 7, A-D). We quantified the localization by counting the PSCs associated with 3 distinct mesenchymal locations: the crypt base (adjacent to proliferative ColEPs), the mid-crypt (adjacent to postmitotic epithelial cells), and the crypt surface (adjacent to terminally differentiated epithelial cells). We found that untreat-

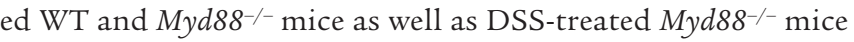
all showed the same distribution of PSCs, with the majority of the cells present in the mid-crypt zone. However, DSS-treated WT mice showed a statistically significant redistribution of cells from the upper and mid-crypt zones to the crypt base associated zone (Figure 7F; $P<0.05$ for each compartment, compared with untreated WT; $n=6-8$ mice/group). The fractional representation of PSCs in the crypt base nearly doubled with DSS treatment in WT mice. The percentage of PSCs in both the upper and middle zones showed statistically significant decreases $(P<0.05$; Figure 7F).

The Myd88-dependent change in the distribution of PSC-positive cells during injury requires leukocytes. Adoptive transfer of peripheral blood leukocytes from $\mathrm{Rag} 1^{-/-}$mice into $\mathrm{Myd} 88^{-/-}$mice during the course of DSS treatment rescued the epithelial defect in rectal crypts (18.5 \pm 4.9 M-phase cells/100 crypts and $34.8 \pm 2.0$ cells/crypt; $n=6$ mice; compare with Figure 1). The number of PSCs in the adoptively transferred $M y d 88^{-/}$DSS-treated mice was similar to those in all other groups (Figure 7E), and the distribution of PSCs was similar to that in WT DSS-treated mice (Figure 7F). We con- 

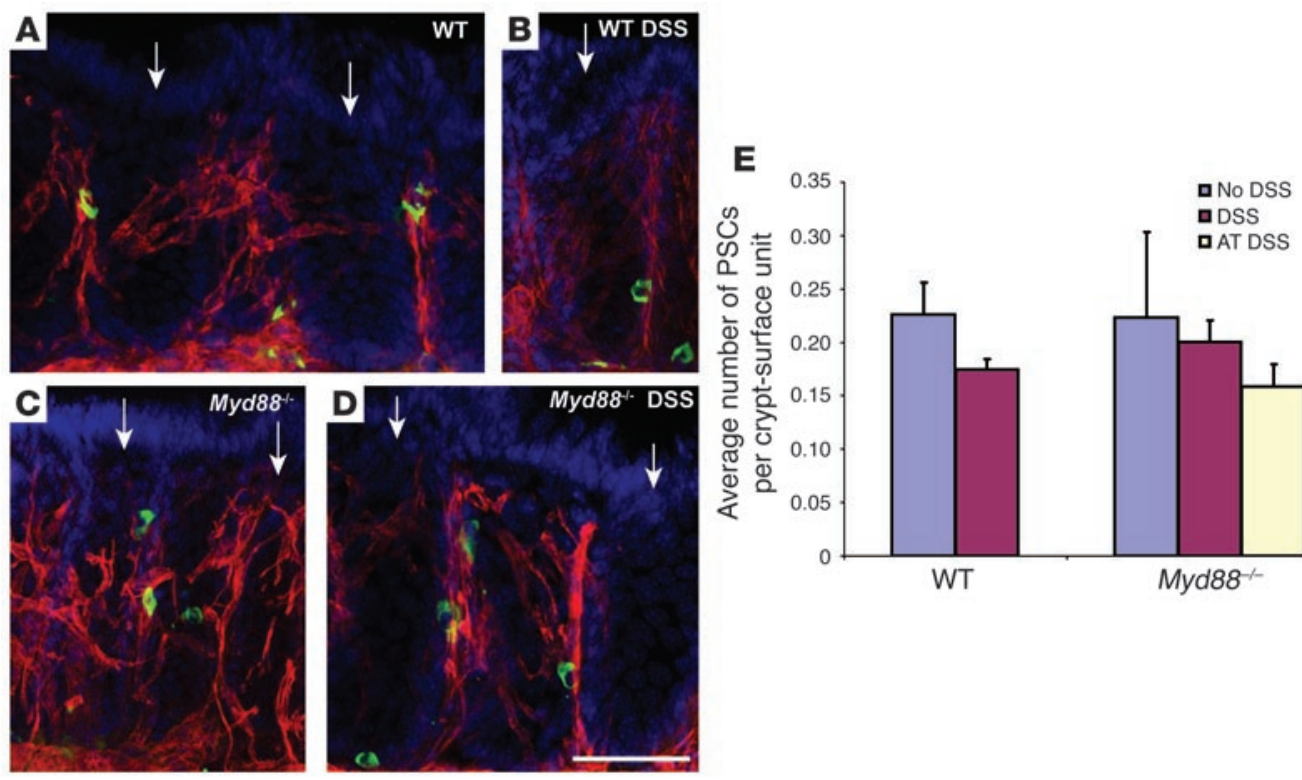

$\mathbf{F}$
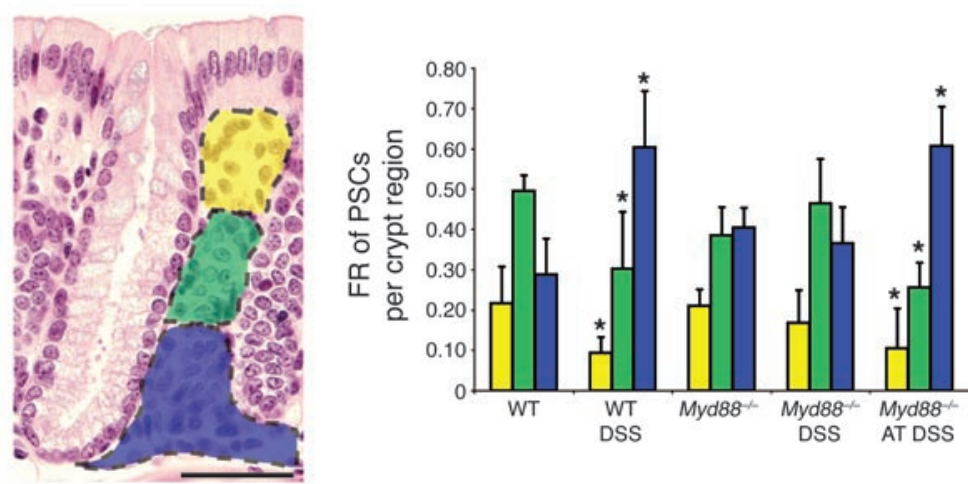

Figure 7

Myd88-dependent alteration of PSC distribution toward the crypt base niche in response to DSS-mediated injury. (A-D) Double-labeled 60- $\mu \mathrm{m}$ rectal sections immunofluorescently stained with Zenon Alexa Fluor 488-labeled anti-Ptgs2 Ig (PSCs, green), Cy3-labeled anti- $\alpha$-SMA Ig (myofibroblasts, red), and bis-benzimide from (A) WT,

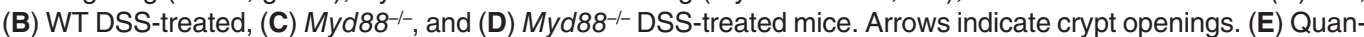
tification of PSCs per crypt-surface unit. No significant differences in the numbers of PSCs were observed when WT and $\mathrm{Myd} 88^{-/-}$DSS-treated mice were compared with their untreated counterparts. AT DSS, $M y d 88^{-/-}$mice treated with DSS and an adoptive transfer of peripheral blood leukocytes from Rag 1-/- mice. (F) Left: Map for PSC quantification in the 3 mesenchymal zones (upper, barrier associated; middle, postmitotic crypt-associated zone; lower, proliferative crypt-associated zone). Right: Quantification of PSC fractional representation (FR) for each zone. An asterisk indicates a significant difference between DSS-treated mice and untreated controls $\left({ }^{\star} P<0.05\right.$;

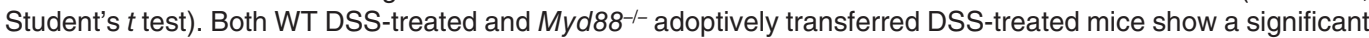
shift in the fractional representation of PSCs toward the crypt base, while similarly treated $M y d 88^{-/}$mice did not show alteration of PSC position. Scale bars: $30 \mu \mathrm{m}$.

clude that an influx of Myd88-competent leukocytes (likely either monocytes or NK cells, as these are the predominant cell types in Rag $1^{-1-}$ blood) enter the gut during DSS-mediated injury and signal either directly or indirectly to the PSCs.

The crypt base-associated PSCs demonstrated a second aspect of their changed location in WT DSS-treated mice, as they appeared to be closely associated with the epithelium. We measured the shortest distance of PSCs to the basal surface of the crypt base epithelium (stained with integrin $\alpha 6$; Figure 8, A-E). This analysis was performed on the crypt base-associated PSCs. We found that this distance was statistically significantly smaller for WT
DSS-treated mice as well as adoptively transferred Myd88-/- DSS-treated mice as compared with all other experimental groups (Figure 8E; 2 - to 3 -fold decrease; $P<0.001$; $n=6-8 \mathrm{mice} /$ group, $n=$ $20-30$ cells/mouse). As a control, we performed a similar evaluation of myofibroblasts (Figure 8, F-J; $n=6-8 \mathrm{mice} /$ group, $n=50$ cells/mouse) and PECAMpositive endothelial cells (average distances/ group $=3.5-4.6 \mathrm{~mm}$ from the base of crypt epithelial cells; data not shown) located in the crypt base niche on adjacent sections of the same mice and found no significant DSS injury- or Myd88dependent alterations in the position of these cells relative to the base of the crypt base epithelium.

Given the recently described role for $\mathrm{PGE}_{2}$ in reinforcing Wnt signaling (28) and our results indicating that the location of the PSCs is critical to maintain proliferation during DSS-mediated injury, we hypothesized that relative transcript abundance in the crypt base (where ColEPs reside) for known Wnt targets would be altered during DSS injury. Possible results were that Wnt signaling would be (a) increased in WT DSStreated ColEPs (relative to WT untreated), (b) diminished in $M y d 88^{-/-}$DSS-treated ColEPs (relative to Myd88-/untreated), or (c) both a and b together. We found that the first scenario occurred. qRT-PCR analysis for matrix metallopeptidase 7 (Mmp7), CD44, cyclin D1 (Ccnd1), and fos-like antigen 1 (Fosl1) using RNAs isolated from LCM-procured crypts bases showed no statistically significant differences in Myd88-/- ColEPs (DSStreated versus untreated). However, each of these transcripts was enriched at least 2.2-fold in WT ColEPs during DSS-mediated injury (Table 2). This result indicates that an increase in Wnt signaling is required to maintain epithelial proliferation during DSS-mediated injury. 
Table 1

Average picograms of $\mathrm{PGE}_{2}$ per milligram of rectal tissue

\begin{tabular}{lcc} 
Condition & Sample 1 & Sample 2 \\
WT & 95.9 & 109.7 \\
WT DSS & 77.0 & 74.6 \\
Myd88-- DSS & 114.7 & 80.1 \\
Ptgs $^{-/}$DSS & 111.4 & 118.5 \\
\hline
\end{tabular}

\section{Discussion}

Preservation of epithelial proliferation is crucial for proper maintenance of the colonic epithelial barrier. ColEPs from mice that lack either Ptgs 2 or Myd88 generate an inappropriate hypoproliferative state when exposed to DSS $(18-21,26)$. In this article, we establish that Ptgs 2 and $\mathrm{PGE}_{2}$ are key downstream targets of Myd88 signaling during DSS-mediated injury that can maintain epithelial proliferation in the face of this injury stimulus. An unanticipated finding was that the mechanistic link was not through the modulation of either Ptgs 2 expression or $\mathrm{PGE}_{2}$ production by Myd88/TLR activity, as we initially hypothesized. Instead, Myd88 signaling controlled the localization of a population of PSCs, a major source of Ptgs 2 in the mouse colon (Figure 9). During injury, PSCs altered their localization to become more enriched within the crypt baseassociated mesenchyme. In addition, the PSCs in this region were more closely associated with the crypt base epithelium, where proliferative ColEPs reside. These 2 PSC redistributions depended on Myd88 signaling and were necessary to maintain epithelial proliferation in the response to injury.

Recent studies have begun to dissect the cellular components and molecular interactions of cellular niches and their associated
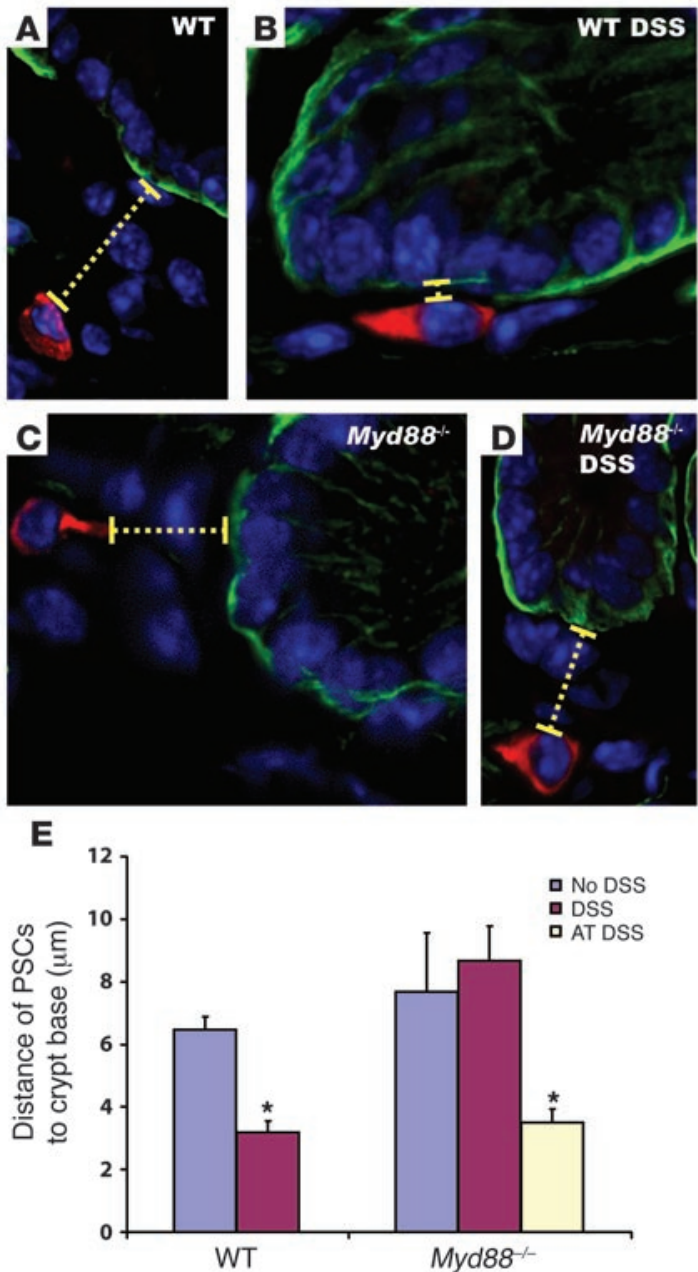
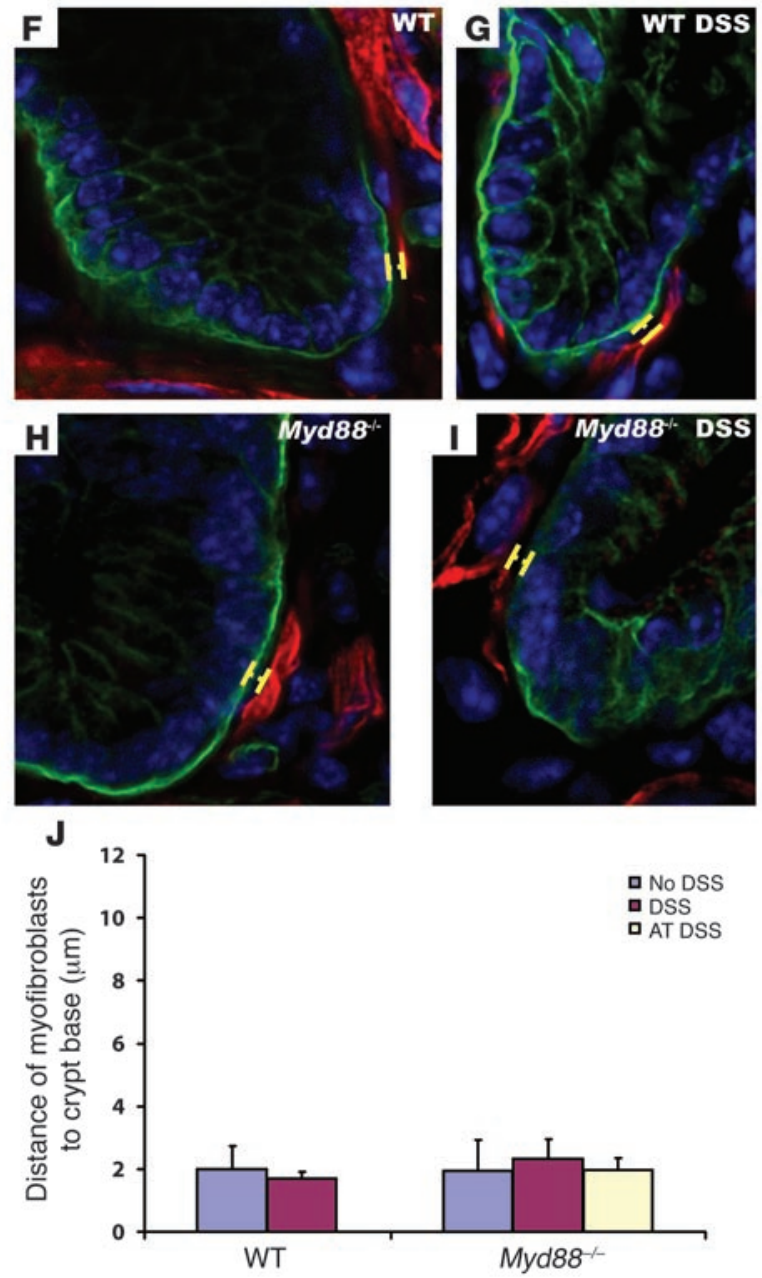

Figure 8

PSCs were closest to the crypt base epithelium in WT DSS-injured mice. (A-D and F-I) Sections of mouse rectums were stained with FITClabeled anti-integrin $\alpha 6 \mathrm{lg}$ (green) to label the basal surface of epithelial cells, bis-benzimide, and either (A-D) Zenon Alexa Fluor 594-labeled anti-Ptgs2 Ig (PSCs, red) or (F-I) Cy3-labeled anti- $\alpha$-SMA Ig (myofibroblasts, red). Samples were taken from (A and F) WT untreated, (B and

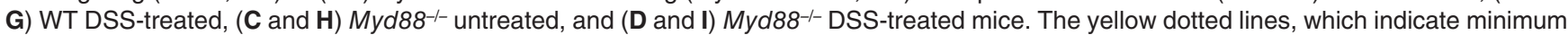
distance from the PSC or myofibroblasts to the base of the crypt epithelium (all views are of the crypt base only), measure $19 \mu \mathrm{m}(\mathbf{A}), 2 \mu \mathrm{m}$ (B), $11 \mu \mathrm{m}(\mathbf{C}), 16 \mu \mathrm{m}(\mathbf{D}), 1.5 \mu \mathrm{m}(\mathbf{F}$ and $\mathbf{I}), 1.3 \mu \mathrm{m}(\mathbf{G})$, and $1.7 \mu \mathrm{m}(\mathbf{H})$. Quantification of distances between the crypt base and (E) PSCs and (J) anti- $\alpha$-SMA-positive myofibroblasts. Mean values \pm SEM were plotted for each group. An asterisk indicates a value that is statistically significantly different from the corresponding untreated control ( ${ }^{\star} P<0.001$; Student's $t$ test). 


\section{Table 2}

Fold difference (as determined by qRT-PCR) of transcripts in the crypt base, comparing DSS-treated mice to untreated counterparts

\begin{tabular}{lcccc} 
Mice & \multicolumn{4}{c}{ Transcripts } \\
& CD44 & Fos/1 & Ccnd1 & Mmp7 \\
WT & $7.1 \pm 1.1$ & $3.6 \pm 0.8$ & $2.4 \pm 0.3$ & $2.2 \pm 0.9$ \\
Myd88--- & $1.3 \pm 0.5$ & $1.1 \pm 0.8$ & $1.1 \pm 0.4$ & $1.2 \pm 0.4$
\end{tabular}

stem and progenitor cells in a variety of mammalian systems such as bone marrow and skin as well as in simpler model organisms such the gonads of Drosophila melanogaster and Caenorhabditis elegans (for reviews, see refs. 13, 14). All of these systems emphasize the close contact of the niche with the target stem cell in order to effect proper communication. This study along, with our previous study (18), emphasizes that during specific perturbations, the colonic niche is not a fixed vessel. One mechanism of injury adjustment is the recruitment of additional cellular constituents. We previously found that activated macrophages, likely recruited from blood monocytes, are one key cell type that performs this function in the intestine (18). PSCs appear to be recruited from a much more local cellular pool that is not expanded during injury and are a second cell type that is mobilized to the crypt niche to become juxtaposed to ColEPs during injury.

Several lines of evidence suggest that epithelial proliferation is preserved in WT mice treated with DSS by the altered distribution of PSCs and their generation of $\mathrm{PGE}_{2}$. First, in the absence of TLR signaling, neither PSC repositioning nor preservation of proliferation during injury occurs. Second, in the absence of Ptgs2, there is no preservation of proliferation. Third, in both

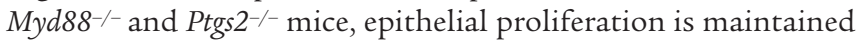
by the administration of a stable analog of $\mathrm{PGE}_{2}$. The key event in preserving epithelial proliferation appears to be the achievement of high levels of $\mathrm{PGE}_{2}$ in the pericryptal niche. $\mathrm{PGE}_{2}$ has a short half-life and acts over short distances $(34,35)$. Therefore, PSCs positioned closer to crypt epithelial cells should increase the local concentration of $\mathrm{PGE}_{2}$. In WT mice, $\mathrm{PGE}_{2}$ levels in the pericryptal region are increased by moving the $\mathrm{PGE}_{2}$-producing cells closer; we

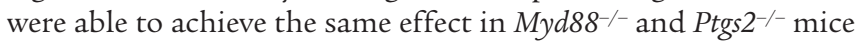
by administering a stable analog of $\mathrm{PGE}_{2}$. The mechanism of the redistribution of the PSCs is unknown, and we hypothesize that this occurs by cell migration in response to either a chemotactic factor or basement membrane modification. It is possible that this same factor could recruit activated macrophages as well.

Total rectal $\mathrm{PGE}_{2}$ content is similar in all the conditions tested, including in DSS-treated Ptgs $2^{-/}$mice, suggesting that the majority of the rectal $\mathrm{PGE}_{2}$ is synthesized through Ptgs1. These data are consistent with previous studies demonstrating the presence of large numbers of Ptgs1-expressing cells and small numbers of Ptgs2-expressing cells in the gastrointestinal tract (22-24) and that the intestinal $\mathrm{PGE}_{2}$ levels are decreased in Ptgs $1^{-/-}$mice (26). It is important to note that the rectal $\mathrm{PGE}_{2}$ level represents the integration of numerous $\mathrm{PGE}_{2}$ microenvironments within this tissue. Current methods do not allow measurement of $\mathrm{PGE}_{2} \mathrm{lev}$ els in specific microenvironments. It is reasonable to assume that although Ptgs2 does not contribute extensively to total rectal
$\mathrm{PGE}_{2}$ levels, it does contribute significantly to $\mathrm{PGE}_{2}$ synthesis in the immediate neighborhood of PSCs.

In these studies, epithelial proliferation was maintained during DSS-mediated injury by repositioning PSCs without any increase in Ptgs2 expression or $\mathrm{PGE}_{2}$ production. Ex vivo, TLR ligands given in pharmacologic quantities can induce Ptgs 2 expression in cells that do not constitutively express Ptgs 2 , such as macrophages and myofibroblasts $(36,37)$. Increased Ptgs 2 expression is commonly associated with markedly increased $\mathrm{PGE}_{2}$ production. Here, in response to the modest level of injury seen in DSS colitis, TLR signaling induces the repositioning of PSCs without (a) inducing higher levels of Ptgs 2 expression in those cells, (b) inducing Ptgs2 expression in cells that do not normally express Ptgs2, or (c) increasing overall $\mathrm{PGE}_{2}$ production in the colon. The importance of the positioning of PSCs to locally provide $\mathrm{PGE}_{2}$ to ColEPs during injury is supported by a recently published study that shows $\mathrm{PGE}_{2}$ affects epithelial proliferation by enhancing Wnt signaling in colonic tumor cells (25). Importantly, loss of Wnt signaling in a normal mouse creates

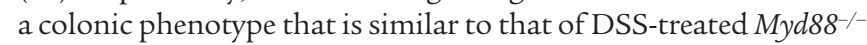
mice (12). Thus, PSCs may be mobile elements that can support critical growth factor pathways during certain types of damage.

These findings may have applications for injury in other organs outside of the gut. Ptgs 2 and $\mathrm{PGE}_{2}$ play positive roles in wound response and healing in bone marrow injured by chemotherapeutic drugs and in bone injured by fractures $(38,39)$. Myd88 signaling has recently been shown to be required for resistance of lung epithelial cells to bleomycin injury (40). Our studies suggest that the linkage of Myd88 and Ptgs2 signaling may occur in these other tissues. If so, then the role of the positioning of PSCs with regard to tissue stem cells would be of interest. The advantage of the colonic injury system as a model for uncovering and quantifying cellular

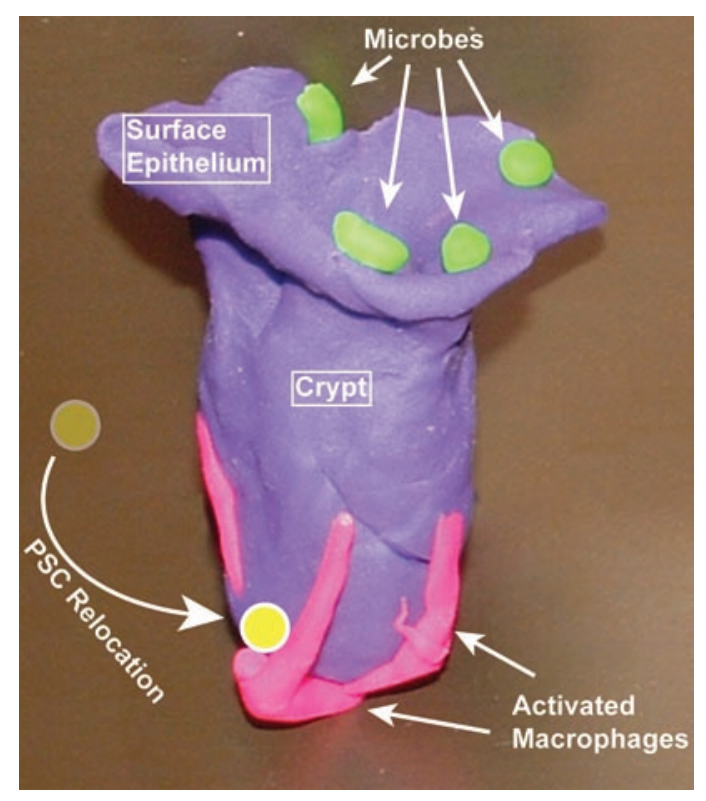

\section{Figure 9}

Model of the key cells that maintain ColEP proliferation and crypt morphology during DSS-mediated injury. Required cellular elements in this system are luminal microbes (green), activated macrophages that contain long cellular processes near the crypt base (pink), and PSCs (yellow) that relocate to the crypt base near the activated macrophages and adjacent to the ColEPs. 
redistribution is the well-defined microanatomy and organization of the epithelium and its associated mesenchyme.

In conclusion, this model supports the concept that the niche established by pericryptal cells is important in regulating epithelial cell proliferation in the colon. It also supports the suggestion that $\mathrm{PGE}_{2}$ promotes epithelial proliferation and epithelial homeostasis in the face of injury. One aspect of the model that we believe to be novel is the demonstration that a population of PSCs is repositioned in response to injury and promotes epithelial homeostasis through $\mathrm{PGE}_{2}$ production. Thus, the pericryptal niche is even more plastic than previously thought, and the cellular composition of the niche is modulated in response to injury. The second aspect of this model that we believe to be novel is the demonstration that the repositioning of the PSCs is Myd88 dependent. This observation provides a mechanism for the more severe DSS-induced colitis seen

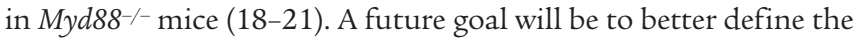
PSC cell type and its properties in the intestine and extraintestinal sites. The challenge will be to isolate these mesenchymal cells without inducing Ptgs 2 in additional cell populations.

\section{Methods}

Mice. All experiments involving animals were approved by the Washington University School of Medicine Animal Studies Committee. All mice were maintained in a specific pathogen-free barrier facility under a strict 12-hour light/dark cycle and fed the same autoclaved chow diet (B\&K Universal Ltd.) as was fed to germ-free counterparts that were used in our previous experiments (18). Adult (6- to 10-week-old) male mice were used

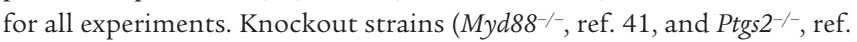
42) were backcrossed more than 10 generations on a B6 background (The Jackson Laboratory). WT controls were the parental B6 strain.

A $2.5 \%$ aqueous solution of DSS (TdB Consultancy) was passed through a $0.22-\mu \mathrm{m}$ cellulose acetate filter and then administered to mice for 7 days as drinking water. Control groups of mice received water alone. $\mathrm{dmPGE}_{2}$ (Cayman Chemical) was dissolved in ethanol $(10 \mathrm{mg} / \mathrm{ml})$ and diluted into sterile $5 \%$ sodium bicarbonate immediately before use $(2 \mu \mathrm{g} / \mathrm{ml})$. Each mouse received i.p. injections of this solution twice per day $(10 \mu \mathrm{g} / \mathrm{kg}$ body weight) for 7 days, concurrent with DSS treatment.

For adoptive transfer experiments, peripheral blood from B6 male donor Rag $1^{-/-}$mice was collected via the posterior vena cava and placed in a heparinized tube. To purify leukocytes, the blood was underlaid with Lympholyte (Cedarlane Laboratories Ltd.) in a 3:2 ratio and subjected to centrifugation $\left(900 \mathrm{~g}, 20\right.$ minutes, $\left.24^{\circ} \mathrm{C}\right)$. The buffy coat was collected and washed with sterile PBS, and then centrifuged $\left(325 \mathrm{~g}, 10\right.$ minutes, $\left.24^{\circ} \mathrm{C}\right)$. The cell pellet was collected and then suspended in a solution containing $0.1 \%$ BSA in sterile PBS. Acceptor mice were anesthetized with a solution consisting of $87 \mathrm{mg}$ ketamine/ $\mathrm{kg}$ body weight and $13 \mathrm{mg}$ xylazine $/ \mathrm{kg}$ body weight. Purified leukocytes were retroorbitally injected $\left(15 \times 10^{6}\right.$ cells/injection in $100 \mu \mathrm{l}$ of $0.1 \%$ BSA in PBS). Injections were performed on 3 consecutive days during DSS treatment (days 4, 5, and 6). Mice were then sacrificed on day 7, and their colons were collected, fixed in Bouin fixative, and blocked in paraffin for histological analysis.

Histochemical and immunohistochemical analyses. All analysis was performed on samples from the rectum (see Figure 1A for specific region). For morphometric analysis and a subset of immunohistochemical studies, colons were dissected, placed in Bouin fixative for 4 hours at $24^{\circ} \mathrm{C}$, and embedded in $2 \%$ agar for routine paraffin processing. Serial 5 - $\mu \mathrm{m}$-thick sections were cut perpendicular to the crypt-surface epithelial cuff axis and parallel to the cephalocaudal axis. Sections were stained with H\&E or PAS/AB.

For a subset of immunohistochemical studies, cycling cells were labeled in S phase by i.p. injection of a solution containing BrdU $(120 \mathrm{mg} / \mathrm{kg})$ and
5 '-fluor-2'-deoxyuridine (12 mg/ml; Sigma-Aldrich) 1 hour prior to sacrifice. S-phase cells were identified in tissue sections using goat anti-BrdU Ig (30).

For a subset of immunohistochemical studies, colons were dissected as described above and were fixed for 15 minutes at $24^{\circ} \mathrm{C}$ in $1 \%$ paraformaldehyde, $75 \mathrm{mM}$ lysine, $75 \mathrm{mM} \mathrm{NaPO}_{4}$, $\mathrm{pH}$ 7.4. The specimens were washed 3 times for 5 minutes in PBS, pH 7.4, $5 \%$ sucrose at $24^{\circ} \mathrm{C}$, and were frozen in Optimal Cutting Temperature compound (Tissue-Tek) in flat sheets to optimize orientation. For direct immunofluorescence, either 5 - or 60- $\mu \mathrm{m}$-thick sections were additionally postfixed in methanol (5 minutes at $-20^{\circ} \mathrm{C}$ ) and were blocked in $1 \% \mathrm{BSA} / 0.2 \%$ nonfat dry milk in PBS for 15 minutes at $24^{\circ} \mathrm{C}$. Sections were stained with Alexa Fluor 488 -labeled Armenian hamster mAbs against CD3ع (T cells; clone 145-2C11; BD Biosciences - Pharmingen; 1:50 dilution), FITC-labeled rat mAbs against B220 (B cells; clone RA3-6B2; BD Biosciences - Pharmingen; 1:50), Alexa Fluor 488-labeled rat anti-mouse F4/80 (macrophages; clone CI:A3-1; CALTAG Laboratories; 1:50), FITC-labeled rat anti-mouse CD45 (leukocyte common antigen, Ly-5; clone 30-F11; BD Biosciences - Pharmingen; 1:100), FITClabeled rat anti-mouse CD11c (DCs; clone HL3; 1:50); PE-labeled rat CD70 (clone FR70; BioLegend; 1:100), Cy3-labeled mouse anti- $\alpha$-SMA (myofibroblasts; clone 1a4; Sigma-Aldrich; 1:2,000), rabbit neuron $\beta$-tubulin (ganglion cells; CRP Inc.; 1:1,000), Gr-1 (neutrophils and eosinophils; clone RB6-8C5; BD Biosciences - Pharmingen; 1:100), rat anti-mouse CD31 (endothelial cells; clone MEC13.3, BD Biosciences - Pharmingen; 1:100), rat IgG2a anti-human/anti-mouse CD49f (integrin $\alpha 6$; clone NKI-GoH3; Chemicon International), rat IgG2b anti-mouse CD44 (clone IM7; BD Biosciences - Pharmingen), rabbit anti-pan laminin (Sigma-Aldrich), rat IgG2a antihuman prolyl 4-hydroxylase (clone ER-TR7; BMA Biomedicals), rabbit antihuman S100 A4 ab-8 (FSP1; Lab Vision Corp.), and mouse IgG1 anti-Ptgs2 $\mathrm{mAb}$ (clone 33; BD Biosciences; 1:50). The Zenon Antibody Labeling system (Invitrogen) was utilized for labeling rabbit antibodies and mouse mAbs according to the manufacturer's instructions. Antibodies against insulin were used as a negative control for mouse IgG1 (clone Z006; Zymed).

Sections were viewed with a Zeiss Axiovert 200 with AxioCam MRm camera and with ApoTome optical sectioning slider. Two-dimensional rendering of 3-dimensional images (for $60-\mu \mathrm{m}$ sections) was performed using Adobe Photoshop CS2 to compile stacked images at 3- $\mu \mathrm{m}$ intervals.

$P G E_{2}$ assay. Lipids were extracted by homogenizing flash-frozen tissue in $70 \%$ cold ethanol, $30 \%$ monobasic sodium phosphate $(0.1 \mathrm{M}), \mathrm{pH} 4.0$, followed by shaking incubation for 30 minutes at $24^{\circ} \mathrm{C}$. Homogenates were centrifuged at $1,000 \mathrm{~g}$ for 10 minutes. Triplicate aliquots of supernatant were dried under nitrogen, and a $\mathrm{PGE}_{2}$ concentration was determined by ELISA (Cayman Chemical).

$L C M$ and $q R T-P C R$ analysis. Colons from untreated and DSS-treated mice were prepared for LCM using protocols described in ref. 31. The lamina propria mesenchyme and epithelium were separately harvested using a PixCell IIe System and CapSure HS LCM caps (Arcturus; $n=3$ mice per experimental group; 10,000 cells harvested/compartment/mouse). Total cellular RNA was extracted (PicoPure RNA Isolation kit; Arcturus) and its quality evaluated using an Agilent Technologies 2100 Bioanalyzer. Random primed cDNA synthesis was used as a template for qRT-PCR that was performed in triplicate for each biologic sample using SYBR Green Master Mix (Stratagene; all reagents from Invitrogen). The $C_{T}$ for each amplicon was determined as the PCR cycle at which the fluorescence intensity crosses a user-established threshold. The following primers were used: E-cadherin (5'-GTCAACACCTACAACGCTGCC; 5'-GTTGTGCTCAAGCCTTCGC), vimentin (5'-CTTGAACGGAAAGTGGAATCCT and 5'-GTCAGGCTTGGAAACGTCC), $18 \mathrm{~S}$ (5'-CATTCGAACGTCTGCCCTATC and 5' - CCTGTGCCTTCCTTGGA), Ptgs2 (5'-TGCCTGGTCTGATGATGTATG and 5'-GGGGTGCCAGTGATAGAGTG), PECAM (5'-TGCACCCATCACTTACCACC and 5'-CTTCATCCACCGGGGCTATC), 
CD11c (5'-GCAGGAGTGTCCAAAGCAAGA and 5'-CGTGTGCTAGGTCTCTGAAGC), F4/80 (5'-GCTGCCAAGTTAATGGACTCA and $5^{\prime}$-ACTGTGGAAAGCACCATGTTAG), Iigp1 (5'-CAGGACATCCGCCTTAACTGT and 5' - AGGAAGTAAGTACCCATTAGCCA), Reg3g (5'-CTATGAACCCAACAGAGGTGGA and 5'-CACTTCAGAAATCCTGAGGCTC), Indo (5'-ATTGGTGGAAATCGCAGCTTC and 5'-ACAAAGTCACGCATCCTCTTAAA), Mmp7 (5'-ACTTCAGACTTACCTCGGATCG and 5'-TCCCCCAACTAACCCTCTTGA), Fosl (5'-GCGGAGACCGACAAATTGGA and $5^{\prime}$-TTCTGGGATTTTGCAGATGGG), Ccnd1 (5'-TTGTGCATCTACACTGACAACTC and 5'-AGGGTGGGTTGGAAATGAACT), and CD44 (5'-TCCAGTCATAGTACAACCCTTCA and 5'-GCTCTTAGTGCTAGATGGCAGA).

Statistics. For calculations to determine statistical significance, a 1tailed, homoscedastic Student's $t$ test was used. SEMs were taken from calculations of respective SDs. All such calculations were performed using Microsoft Excel 2003.

1. Savage, D.C. 1977. Microbial ecology of the gastrointestinal tract. Annu. Rev. Microbiol. 31:107-133.

2. Eckburg, P.B., et al. 2005. Diversity of the human intestinal microbial flora. Science. 308:1635-1638.

3. Ley, R.E., etal. 2005. Obesityalters gut microbialecology. Proc. Natl. Acad. Sci. U. S. A. 102:11070-11075.

4. Stappenbeck, T.S., Hooper, L.V., and Gordon, J.I. 2002. Developmental regulation of intestinal vasculogenesis by indigenous microbes via Paneth cells. Proc. Natl. Acad. Sci. U. S. A. 99:15451-15456

5. Dotan, I., and Mayer, L. 2003. Intestinal immunity. In Microbial pathogenesis and the intestinal epithelial cell. G.A. Hecht, editor. ASM Press. Washington, DC, USA. 43-60.

6. Chang, W.W., and Leblond, C.P. 1971. Renewal of the epithelium in the descending colon of the mouse. Am. J. Anat. 131:73-99.

7. Chang, W.W., and Nadler, N.J. 1975. Renewal of the epithelium in the descending colon of the mouse. Am. J. Anat. 144:39-56.

8. Pinto, D., Gregorieff, A., Begthel, H., and Clevers, H. 2003. Canonical Wnt signals are essential for homeostasis of the intestinal epithelium. Genes Dev. 17:1709-1713.

9. Van Es, J.H., et al. 2005. Notch/gamma-secretase inhibition turns proliferative cells in intestinal crypts and adenomas into goblet cells. Nature. 435:959-963.

10. Van den Brink, G.R., et al. 2004. Indian Hedgehog is an antagonist of Wnt signaling in colonic epithelial cell differentiation. Nat. Genet. 36:277-282.

11. Haramis, A.P., et al. 2004. De novo crypt formation and juvenile polyposis on BMP inhibition in mouse intestine. Science. 303:1684-1686.

12. Kuhnert, F., et al. 2004. Essential requirement for Wht signaling in proliferation of adult small intestine and colon revealed by adenoviral expression of Dickkopf-1. Proc. Natl. Acad. Sci. U. S. A. 101:266-271.

13. Spradling, A., Drummond-Barbosa, D., and Kai, T. 2001. Stem cells find their niche. Nature. 414:12-18.

14. Fuchs, E., Tumbar, T., and Guasch, G. 2004. Socializing with the neighbors: stem cells and their niche. Cell. 116:769-778.

15. Adegboyega, P.A., Mifflin, R.C., DiMari, J.F., Saada, J.I., and Powell, D.W. 2002. Immunohistochemical study of myofibroblasts in normal colonic mucosa, hyperplastic polyps, and adenomatous colorectal polyps. Arch. Pathol. Lab. Med. 126:829-836.

16. Marsh, M.N., and Trier, J.S. 1974. Morphology and cell proliferation of subepithelial fibroblasts in adult mouse jejunum. II. Radioautographic studies. Gastroenterology. 67:636-645.

\section{Acknowledgments}

This work was funded by the NIH (DK02954, DK33165, DK55753, and DK66220), the Crohn's and Colitis Foundation of America, the Pew Scholars Program in the Biomedical Sciences, and the Washington University Digestive Disease Research Core (NIH P30-DK52574).

Received for publication May 19, 2006, and accepted in revised form October 3, 2006.

Address correspondence to: Thaddeus S. Stappenbeck, Department of Pathology and Immunology, Washington University School of Medicine, Box 8118, 660 S. Euclid Avenue, St. Louis, Missouri 63110, USA. Phone: (314) 362-4214; Fax: (314) 362-7487; E-mail: stappenb@pathology.wustl.edu.
17. Paris, F., et al. 2001. Endothelial apoptosis as the primary lesion initiating intestinal radiation damage in mice. Science. 293:293-297.

18. Pull, S.L., Doherty, J.M., Mills, J.C., Gordon, J.I., and Stappenbeck, T.S. 2005. Activated macrophages are an adaptive element of the colonic epithelial progenitor niche necessary for regenerative responses to injury. Proc. Natl. Acad. Sci. U. S. A. 102:99-104.

19. Rakoff-Nahoum, S., Paglino, J., Eslami-Varzaneh, F., Edberg, S., and Medzhitov, R. 2004. Recognition of commensal microflora by TLRs is required for intestinal homeostasis. Cell. 118:229-241.

20. Araki, A., et al. 2005. MyD88-deficient mice develop severe intestinal inflammation in dextran sodium sulfate colitis. J. Gastroenterol. 40:16-23.

21. Fukata, M., et al. 2005. TLR-4 is required for intestinal response to epithelial injury and limiting bacterial translocation in a murine model of acute colitis. Am. J. Physiol. Gastrointest. Liver Physiol. 288:G1055-G1065.

22. Newberry, R.D., McDonough, J.S., Stenson, W.F., and Lorenz, R.G. 2001. Spontaneous and continuous cyclooxygenase-2-dependent PGE2 production by stromal cells in the murine small intestine lamina propria: directing the tone of the intestinal immune response. J. Immunol. 166:4465-4472.

23. Riehl, T.E., Newberry, R.D., Lorenz, R.G., and Stenson, W.F. 2004. TNFR1 mediates the radioprotective effects of lipopolysaccharide in the mouse intestine. Am. J. Physiol. Gastrointest. Liver Physiol. 286:G166-G173.

24. Tessner, T.G., Cohn, S.M., Schloemann, S., and Stenson, W.F. 1998. PGs prevent decreased epithelial cell proliferation associated with dextran sodium sulfate injury in mice. Gastroenterology. 115:874-882

25. Wang, D., Mann, J.R, and DuBois, R.N. 2005. The role of prostglandins and other eicosanoids in the gastrointestinal tract. Gastroenterology. 128:1445-1461.

26. Morteau, O., et al. 2000. Impaired mucosal defense to acute colonic injury in mice lacking cyclooxygenase-1 or cyclooxygenase-2. J. Clin. Invest. 105:469-478.

27. Tessner, T.G., Muhale, F., Riehl, T.E., Anant, S., and Stenson, W.F. 2004. PGE2 reduces radiation-induced epithelial apoptosis through a mechanism involving AKT activation and bax translocation. J. Clin. Invest. 114:1676-1685. doi:10.1172/JCI200422218.

28. Castellone, M.D., Teramoto, H., Williams, B.O., Druey, K.M., and Gutkind, J.S. 2005. PGE2 promotes colon cancer cell growth through a Gs-axinbeta-catenin signaling axis. Science. 310:1504-1510.
29. Rosai, J. 1996. Special techniques in surgical pathology. In Ackerman's surgical pathology. MosbyYear Book Inc. St. Louis, Missouri, USA. 29-31.

30. Stappenbeck, T.S., Mills, J.C., and Gordon, J.I. 2003. Molecular features of adult mouse small intestinal epithelial progenitors. Proc. Natl. Acad. Sci. U. S. A. 100:1004-1009.

31. Stappenbeck, T.S., Hooper, L.V., Manchester, J.K., Wong, M.H., and Gordon, J.I. 2002. Laser capture microdissection of the mouse intestine: characterizing mRNA and protein expression, and profiling intermediary metabolism in specified cell populations. Methods Enzymol. 356:167-196.

32. Sonoshita, M., Takaku, K., Oshima, M., Sigihara, K., and Taketo, M. 2002. Cyclooxygenase- 2 expression in fibroblasts and endothelial cells of intestinal polyps. Cancer Res. 62:6846-6849.

33. Laouar, A., et al. 2005. CD70+ antigen-presenting cells control the proliferation and differentiation of $\mathrm{T}$ cells in the intestinal mucosa. Nat. Immunol. 6:698-706.

34. Nomura, T., et al. 2005. Prostaglandin signaling in the renal collecting duct. J. Biol. Chem. 280:28424-28429.

35. Forstermann, U., and Neufang, B. 1983. Elimination from circulation of cats of 6-keto-PGE1 compared with PGE2 and PGI2. J. Pharm. Pharmacol. 35:724-728.

36. Lang, R., Patel, D., Morris, J.J., Rutschman, R.L., and Murray, P.J. 2002. Shaping gene expression in activated and resting primary macrophages by IL-10. J. Immunol. 169:2253-2263.

37. Zhang, Z., et al. 2005. Interleukin-17 and lipopolysaccharides synergistically induce cyclooxygenase2 expression in human intestinal myofibroblasts. J. Gastroenterol. Hepatol. 20:619-627.

38. Lorenz, M., et al. 1999. Cyclooxygenase- 2 is essential for normal recovery from 5-fluorouracil-induced myelotoxicity in mice. Exp. Hematol. 27:1494-1502.

39. Zhang, X., et al. 2002. Cyclooxygenase- 2 regulates mesenchymal cell differentiation into the osteoblast lineage and is critically involved in bone repair. J. Clin. Invest. 109:1405-1415. doi:10.1172/ JCI200215681.

40. Jiang, D., et al. 2005. Regulation of lung injury and repair by TLRs and hyaluronan. Nat. Med. 11:1173-1179.

41. Adachi, O., et al. 1998. Targeted disruption of the MyD88 gene results in loss of IL-1- and IL-18-mediated function. Immunity. 9:143-150.

42. Morham, S.G., et al. 1995. Ptgs2 gene disruption causes severe renal pathology in the mouse. Cell. 83:473-482. 